\title{
Naringin Protects Against High Glucose- Induced Human Endothelial Cell Injury Via Antioxidation and CX3CL1 Downregulation
}

\author{
Guilin Lia Yurong Xu $\mathrm{Xu}^{\mathrm{a}}$ Xuan Sheng ${ }^{\mathrm{a}}$ Hua Liu ${ }^{\mathrm{c}}$ Jingjing Guo Jiayue Wang ${ }^{\mathrm{d}}$ \\ Qi Zhonge Huaide Jiang ${ }^{a}$ Chaoran Zhenga ${ }^{a}$ Mengxia Tan ${ }^{a}$ Shenqiang Rao ${ }^{a}$ \\ Yanling Yu ${ }^{f}$ Yun Gao Guodong Lia Shangdong Liang ${ }^{a}$ Gaochun Zhu ${ }^{\mathrm{b}}$ \\ aDepartment of Physiology, Medical College of Nanchang University, ${ }^{b}$ Department of Anatomy, \\ Medical College of Nanchang University, c Obstetrics and Gynecology Department of First Affiliated \\ Hospital, Nanchang University, ${ }^{d}$ Department of the First Clinical, Medical College of Nanchang \\ University, ${ }^{e}$ Queen Mary School, Nanchang University, ${ }^{f}$ School of Foreign Language of Nanchang \\ University, Nanchang, P. R. China
}

\section{Key Words}

Naringin • HUVEC • CX3CL1 • ROS • Mitochondria • Oxygen consumption rate

\begin{abstract}
Background/Aims: The induction of endothelial injury by hyperglycemia in diabetes has been widely accepted. Naringin is a bio-flavonoid. Some studies showed that naringin alleviates diabetic complications, but the exact mechanisms by which naringin improves diabetic anomalies are not yet fully understood. The aim of this research was to study the protective effect of naringin on high glucose-induced injury of human umbilical vein endothelial cells (HUVECs). Methods: HUVECs were cultured with or without high glucose in the absence or presence of naringin for 5 days. The expression of CX3CL1 was determined by quantitative real-time RT-PCR ( $q P C R$ ) and western blot. The cellular bioenergetic analysis oxygen consumption rate (OCR) was measured with a Seahorse Bioscience XF analyzer. Results: The production of reactive oxygen species (ROS), the expression of CX3CL1 and the level of AKT phosphorylation were increased in HUVECs cultured with high glucose compared with controls. However, naringin rescued these increases in ROS production, CX3CL1 expression and AKT phosphorylation. Nitric oxide (NO) production and OCR were lower in the high glucose group, and naringin restored the changes induced by high glucose. Molecular docking results suggested that Naringin might interact with the CX3CL1 protein. Conclusion: Naringin protects HUVECs from high-glucose-induced damage through its antioxidant properties by downregulating CX3CL1 and by improving mitochondrial function.
\end{abstract}




\section{Cellular Physiology Cell Physiol Biochem 2017;42:2540-2551 \begin{tabular}{l|l|l} 
and Biochemistry Published online: August 23, 2017 & $\begin{array}{l}\text { (c) } 2017 \text { The Author(s). Published by S. Karger AG, Basel } \\
\text { www.karger.com/cpb }\end{array}$
\end{tabular} \\ Li et al.: Naringin Protects Huvecs from HG-Induced}

\section{Introduction}

Diabetes mellitus (DM) is a severe metabolic disease that constitutes a major global health problem. The most important complication of type $2 \mathrm{DM}$ is cardiovascular disease. Vascular endothelial cells play a very important role in the maintenance of cardiovascular homeostasis. Oxidative reactions lead to endothelial dysfunction [1, 2]. Hyperglycemia can increase reactive oxygen species (ROS) production in the mitochondrial electron transport chain, a process that is involved in the pathogenesis of diabetic cardiovascular complications $[3,4]$. Oxidative stress is a result of the excessive production of ROS, and combined with an attenuated antioxidant defense system of the cells, leads to the cellular environment being overwhelmed by an oxidative environment [2]. High glucose causes oxidative stress, and the increase in reactive oxygen species affects mitochondrial oxidative metabolism $[4,5]$. Inflammation and vessel blockage may be initiated by active compounds released by the injured endothelium. Dysfunction and damage of endothelial cells play a crucial role in the pathology of diabetic cardiovascular complications [6]. Our previous results from the RT ${ }^{2}$ Profiler ${ }^{\mathrm{TM}}$ PCR Array of human endothelial cell biology showed that CX3CL1 was upregulated in HUVECs after chronic culture with high glucose [7]. CX3CL1, chemokine (C-X3-C motif) ligand 1, also known as fractalkine, is a large cytokine protein of 373 amino acids. CX3CR1 is the sole receptor for CX3CL1. CX3CL1 elicits adhesive and migratory functions by interacting with the chemokine receptor CX3CR1. The CX3CL1 / CX3CR1 axis has been implicated in the pathogenesis of inflammatory diseases such as atherosclerosis, renal fibrosis and rheumatoid arthritis. Elevated plasma levels of CX3CL1 were described for several classical risk factors of cardiovascular disease, including diabetes/metabolic syndrome, obesity, hyperlipidemia and hypertension [8].

Traditional Chinese medicine has a long history in the treatment of diabetes. Naringin, also known as 4, 5,7-trihydroxyflavanone-7-rhamnoglucoside, is a bio-flavonoid that is derived from tomatoes, grapefruit, and related citrus fruits. It has been reported to be an antioxidant with potent free radical scavenging properties, with the ability to reduce inflammation, mitigate the effects of hyperglycemia, and inhibit apoptosis [9-11]. Naringin has also been reported to prevent the progression of hyperglycemia by increasing hepatic glycolysis and glycogen concentration, and lowering hepatic gluconeogenesis [12]. This reduces hyperlipidemia and hyperglycemia, mitigating the obesity-related inflammatory state in cats with metabolic syndrome. These effects are due to AMPK stimulation, MAPK pathway blockage and the activation of IRS-1 [13]. More recently, naringin was shown to ameliorate cognitive deficits through a combination of effects on oxidative stress, proinflammatory factors and the PPAR $\gamma$ signaling pathway in type 2 diabetic rats [14]. It has also been shown to alleviate diabetic kidney disease by reducing oxidative stress and inflammation [9]. Some studies have suggested that naringin mitigates cardiac hypertrophy by reducing oxidative stress and inactivating the c-Jun nuclear kinase-1 protein in type I diabetes [11]. However, the exact mechanisms by which naringin improves diabetic anomalies are not yet fully understood. Therefore, the present study investigated the potential protective role of naringin against injuries to vascular endothelial cells under high glucose conditions.

\section{Materials and Methods}

\section{HUVEC culture, MTS assay and BrdU assay}

Human umbilical vein endothelial cells (HUVECs) were obtained from CTCC Bioscience. HUVECs were cultured in RPMI 1640 medium supplemented with 10\% FBS containing penicillin (100 U/mL) and streptomycin $(100 \mu \mathrm{g} / \mathrm{mL})$ in a $37^{\circ} \mathrm{C}$ incubator with $5 \% \mathrm{CO}_{2}$. Cells were cultured with $5.5 \mathrm{mM}$ glucose as a control. Naringin was purchased from the Chinese Food and Drug Verification Institute and met the monomer quality standard set by the National Pharmacopoeia of China.

Cell viability was measured with a Cell Titer $96^{\mathrm{TM}}$ AQueous cell viability assay kit (MTS, Promega). HUVECs were seeded in 96-well tissue culture plates at a density of $5 \times 10^{3}$ cells/well. After the cells were 


\section{Cellular Physiology Cell Physiol Biochem 2017;42:2540-2551 \begin{tabular}{l|l|l} 
and Biochemistry Published online: August 23, 2017 & $\begin{array}{l}\text { C) } 2017 \text { The Author(s). Published by S. Karger AG, Basel } \\
\text { www.karger.com/cpb }\end{array}$ \\
\hline
\end{tabular}}

Li et al.: Naringin Protects Huvecs from HG-Induced

cultured for 5 days, they were transferred into fetal bovine serum-free medium containing 20\% MTS and incubated for $3 \mathrm{~h}$ in $37^{\circ} \mathrm{C}$. Formazan production was measured by absorbance at $490 \mathrm{~nm}$ with a multifunctional plate reader.

Cell proliferation was observed via the BrdU assay. HUVECs were cultured in the presence of BrdU for $2 \mathrm{~h}$, then fixed in ethanol/glycine and processed for BrdU immunocytochemistry according to the manufacturer's instructions.

\section{Quantitative real-time RT-PCR}

Total RNA of HUVECs cultured under different conditions was isolated and reverse transcribed into cDNA. Quantitative real-time RT-PCR using SYBR-green was performed with an ABI 7500 PCR System. The sense and antisense primers were 5'-CCTTGGTTAGGCATTGTGGG-3', 5'-TTGGTGGCTTGATGGTGGAA-3' for CX3CL1; for $\beta$-actin, they were 5'-CAAGAGATGGCCACGGCTGCT-3', 5'-TCCTTCTGCATCCTGTCGGCA-3'. Relative levels of mRNA in each sample were calculated in comparison to the respective levels of $\beta$-actin.

\section{Western blot}

Total protein was extracted from HUVECs, separated by SDS-polyacrylamide gel electrophoresis and transferred to a polyvinylidene fluoride membrane. After blocking for $2 \mathrm{~h}$ at room temperature, the membranes were incubated overnight at $4^{\circ} \mathrm{C}$ with the following primary antibodies: rabbit polyclonal anti-CX3CL1 (1:800), anti-PAKT (1:1000), anti-AKT (1:1000), and monoclonal anti- $\beta$-actin (1:800). The membranes were washed and incubated with secondary antibody (1:2000) at room temperature for $2 \mathrm{~h}$. An ECL chemiluminescence development kit and imaging system were used to obtain images of protein bands. The intensity of bands was analyzed with Image-Pro Plus. Expression level changes of target proteins were calculated by normalizing to the corresponding amount of $\beta$-actin.

\section{Measurement of intracellular reactive oxygen species (ROS)}

The DCF-DA method was used to measure the production of intracellular ROS. HUVECs were grown to confluence on 24-well microplates for 5 days, then incubated with PBS containing $10 \mu \mathrm{M}$ DCFH-DA for 25 min at $37^{\circ} \mathrm{C}$. Afterwards, cells were washed twice with PBS, incubated in RPMI 1640 medium for another $30 \mathrm{~min}$, and washed three times with PBS. A fluorescent plate reader was used to measure fluorescence at excitation and emission wavelengths of 485 and $535 \mathrm{~nm}$, respectively. Each sample was run in triplicate, and the average values were normalized to their respective controls.

\section{Determination of nitric oxide (NO) production}

HUVECs were cultured for 5 days with different treatments in 24-well culture plates. NO production was measured with a nitrate reductase assay kit according to the manufacturer's instructions. The absorbance was measured at $540 \mathrm{~nm}$. The concentration of NO was calculated as follows: concentration of NO $(\mu \mathrm{M})=[$ (absorbance of treated wells-absorbance of blank wells) / (absorbance of standard wellsabsorbance of blank wells) $\times$ standard concentration $(100 \mu \mathrm{M})]$.

\section{Mitochondrial stress testing}

The mitochondrial function of HUVECs was measured in intact cells with the XF24 analyzer from Seahorse Bioscience (Billerica, MA, USA). The oxygen consumption rate (OCR), a measure of mitochondrial respiration, was determined in the presence of specific mitochondrial activators and inhibitors. Basal respiration is predominantly controlled by ATP synthase and proton leak. The addition of oligomycin, an ATP synthase blocker, blocks ATP synthase (ATP production); the residual respiration is due to proton leak, which results in a significant decrease in OCR. Maximum respiratory function (maximal OCR) was induced by the electron transport chain accelerator FCCP (carbonyl cyanide 4-(trifluoromethoxy) phenylhydrazone, also known as a mitochondrial uncoupler), which stimulated a high artificial proton conductance into the membrane. Finally, the electron transport chain inhibitors rotenone (an inhibitor of complex I) and antimycin A (a blocker of complex III) were added to completely inhibit mitochondrial respiration [15].

HUVECs were seeded into Seahorse XF 24-well plates and cultured in a $37^{\circ} \mathrm{C}$ incubator with $5 \% \mathrm{CO}_{2}$. Cell concentrations were allowed to reach $2 \times 10^{5} \mathrm{cells} / \mathrm{ml}$ before analysis. Background wells only contained culture medium. After $12 \mathrm{~h}$, cells were split into different groups. Each well was washed twice with $1 \mathrm{ml}$ of $37^{\circ} \mathrm{C}$ preheated test mitochondria, and $500 \mu \mathrm{l}$ of mitochondrial testing liquid were added. Then, the 


\section{Cellular Physiology Cell Physiol Biochem 2017;42:2540-2551

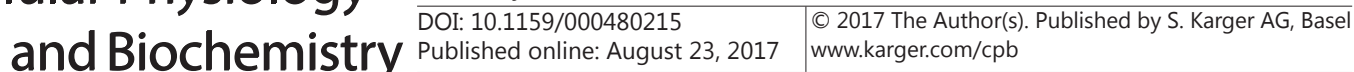 \\ Li et al.: Naringin Protects Huvecs from HG-Induced}

plates were cultured in a $\mathrm{CO}_{2}$-free incubator for $1 \mathrm{~h}$. A probe card was used to add $1 \mathrm{ml}$ of rehydration solution in each well; samples were placed in a $\mathrm{CO}_{2}$-free incubator and cultured for $12-72 \mathrm{~h}$. The probe card was corrected on the machine in approximately $1 \mathrm{~h}$. Oligomycin was added to A wells (at a $1 \mu \mathrm{M}$ final concentration), FCCP was added to B wells (at a $2 \mu \mathrm{M}$ final concentration), and Rotenone/Antimycin A was added to $\mathrm{C}$ wells (at a $0.5 \mu \mathrm{M}$ final concentration) for the seahorse XF24-wells. After calibration, the hydration plate was replaced by the cell plate for measurement on the machine. OCR was normalized to the cell number calculated at the end of the experiments $[16,17]$.

\section{Molecular docking}

Molecular docking computations were performed with AutoDock 4.2 [18]. Molecular docking is a computer simulation approach that attempts to predict the binding mode of a ligand in the active site of a protein. Molecular docking studies mimic the natural interaction of a ligand with the protein. This procedure thus searches for the proper conformation of a ligand in the active site of a protein and uses that conformation for the calculation of molecular descriptors. For each ligand, a number of configurations, called poses, are generated and scored [18]. The score can be calculated as either a free energy of binding, which takes into account solvation and entropy, the enthalpic term of the free energy of binding, or a qualitative shape-based numerical measure. The final top-scoring poses, along with their scores and conformation energies, are written to a database where they are ready for further analysis. Input files of a target protein (4XT3, Pdb ID) and Naringin as the ligand were prepared with Auto Dock Tools (ADT) and Python scripts named prepare_ligand4. py and prepare_receptor4.py, which are associated with the AutoDock4.2 program. The binding pocket position in the target protein was specified with the ADT molecular viewer. The parameters were maintained at their default values. Finally, the output files were viewed with MGLtools1 and PyMol (http://www.pymol.org/). The CX3CL1 structure from Burg et al [19]. was used. The structural formula of naringin was obtained from NCBI (https://pubchem.ncbi.nlm.nih. gov/compound/442428\#section=Top).

\section{Statistical analysis}

All results were expressed as the mean \pm SEM. Statistical analysis was performed with SPSS 11.5. One-factor analysis of variance (ANOVA) was used, followed by post hoc Student's $t$-test. The results were determined to be significant when the $p$ value was less than 0.05 .

\section{Results}

The effects of naringin on HUVEC cell viability

To avoid possible negative effects of naringin on HUVECs, cell viability was assessed with the MTS assay after cells were treated with various concentrations of naringin. The results

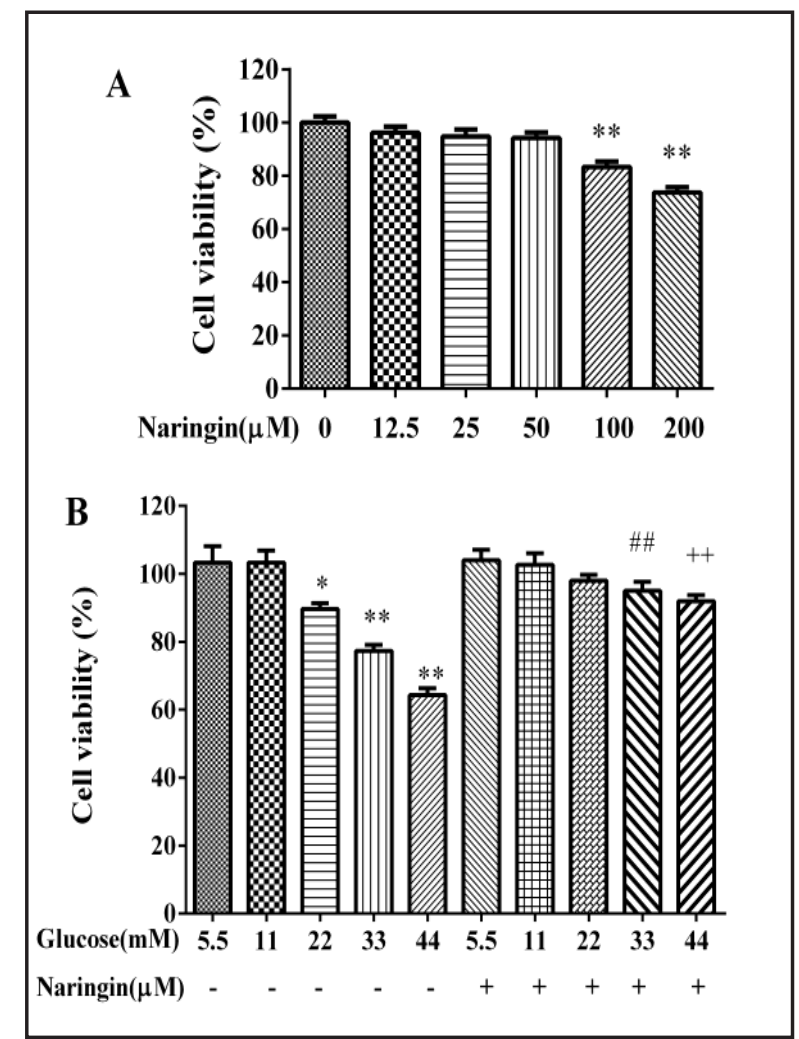

Fig. 1. Effect of naringin on the cell viability of HUVECs. (A) HUVECs had been cultured in 96-well plates with different concentrations of naringin for 5 days, and cell viability (optic density at $490 \mathrm{~nm}$ ) was assessed by MTS after incubation at $37^{\circ} \mathrm{C}$ for $2 \mathrm{~h}$. (B) MTS assays were performed after HUVECs were cultured with control (5.5 mM, Ctrl) or high glucose (11 mM, $22 \mathrm{mM}, 33 \mathrm{mM}$ or $44 \mathrm{mM}, \mathrm{HG}$ ) medium in the absence or presence of $50 \mu \mathrm{M}$ naringin. Values are mean \pm SEM of three separate experiments. ${ }^{*} \mathrm{p}<0.05,{ }^{* *} \mathrm{p}<0.01$ vs. Ctrl group; \#\#p<0.01 vs. $33 \mathrm{mM}$ glucose group, $++\mathrm{p}<0.01$ vs. $44 \mathrm{mM}$ glucose group. 


\section{Cellular Physiology Cell Physiol Biochem 2017;42:2540-2551 \begin{tabular}{ll|l} 
and Biochemistry & $\begin{array}{l}\text { DOI: 10.1159/000480215 } \\
\text { Published online: August 23, } 2017\end{array}$ & $\begin{array}{l}\text { (c) } 2017 \text { The Author(s). Published by S. Karger AG, Basel } \\
\text { www.karger.com/cpb }\end{array}$ \\
\hline
\end{tabular}

indicated that there was no effect on cell viability in the cell culture after exposure to naringin at concentrations of $50 \mu \mathrm{M}$ or less for 5 days (Fig. 1A). The $50 \mu \mathrm{M}$ concentration was used in all experiments described below.

HUVECs had been cultured in control medium containing $5.5 \mathrm{mM}$ glucose (Ctrl) or in high glucose medium (11 $\mathrm{mM}, 22 \mathrm{mM}, 33 \mathrm{mM}$ or $44 \mathrm{mM}, \mathrm{HG}$ ) in the absence or presence of $50 \mu \mathrm{M}$ of naringin for 5 days. Cell viability in cultures treated with $33 \mathrm{mM}$ or $44 \mathrm{mM}$ glucose was significantly reduced, as assessed by the MTS assay $(p<0.01)$. Co-culture with $50 \mu \mathrm{M}$ naringin reversed this high glucose-induced decrease in cell viability (Fig. 1B). The following high glucose experiment used $33 \mathrm{mM}$ glucose.

The effects of naringin on HUVEC cell proliferation

Cell proliferation was assessed by quantifying BrdU incorporation in the cells that were actively synthesizing new DNA. HUVECs had been cultured for 2 days in control medium containing 5.5 $\mathrm{mM}$ glucose or in elevated concentrations of glucose $(33 \mathrm{mM})$ in the presence or absence of $50 \mu \mathrm{M}$ naringin. The result suggested that cell proliferation under high glucose conditions was inhibited compared to the control group, but this phenomenon in HUVECs was completely reversed by co-treatment with $50 \mu \mathrm{M}$ naringin (Fig. 2).

The effect of naringin on CX3CL1 expression

To study the possible mechanism for high glucose-induced negative effects on HUVECs, the expression of CX3CL1 (also called fractalkine), a chemokine involved in inflammation, was examined by real-time PCR and western blot. Real-time RT-PCR revealed that the CX3CL1 mRNA level in the HG group was 2.8-fold higher than that of the control group $(p<0.01)$. To identify its ability to protect against the effects of high glucose, naringin was used to treat HUVECs exposed to high glucose. The expression of CX3CL1 mRNA in the HG group treated with naringin was significantly lower than in the untreated HG group $(p<0.01)$. There was no significant difference in the expression levels of CX3CL1 mRNA among the controls, controls treated with naringin, and the group of samples treated with high glucose and naringin $\left(\mathrm{F}_{(2,24)}=0.892, p>0.05\right)$ (Fig. 3A). Western blots showed that the CX3CL1 protein level in HUVECs cultured with high glucose was 1.7-fold higher than in the controls. In the presence of naringin, high glucose-induced CX3CL1 expression was largely abrogated (Fig. 3B).

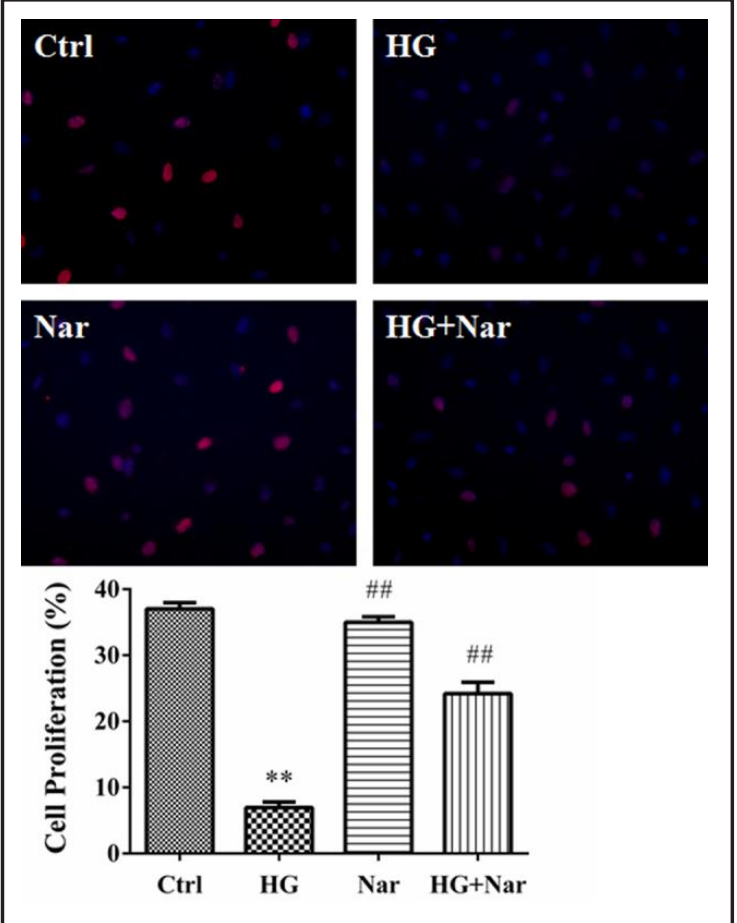

Fig. 2. Effect of naringin on the proliferation of HUVECs. Cell proliferation was measured with BrdU staining. For immunofluorescence, HUVECs had been treated with $33 \mathrm{mM}$ glucose or $50 \mu \mathrm{M}$ naringin for 2 days and then stained for BrdU and counterstained with DAPI (cells were stained blue). BrdU-positive (stained purple) cells were counted in randomly selected microscopic fields (10/replicate) and compared to the total number of cells in these fields. All experiments were conducted in triplicate. Representative photomicrographs from one set of replicates are shown. The cell numbers corresponding to each group were computed and used to calculate cell proliferation (\%) compared to the control group. Magnification: $\times 20{ }^{* *} \mathrm{p}<0.01$ vs. Ctrl group; \#\#p<0.01 vs. HG group. 
Fig. 3. Effects of naringin on CX3CL1 expression in HUVECs at high glucose concentrations. (A) The expression of CX3CL1 mRNA was quantified by realtime RT-PCR analysis and $\beta$-actin was used as an internal control. The results were expressed as the mean \pm SEM of six independent experiments. (B) Detection of CX3CL1 protein by western blot in HUVECs after various treatments. The image is representative of three independent experiments, and the bar histograms show the results of the statistical analysis of the densities of CX3CL1 after normalizing to the amount of $\beta$-actin. ${ }^{* *} \mathrm{p}<0.01$ vs. Ctrl group; $\# \# \mathrm{p}<0.01$ vs. HG group.

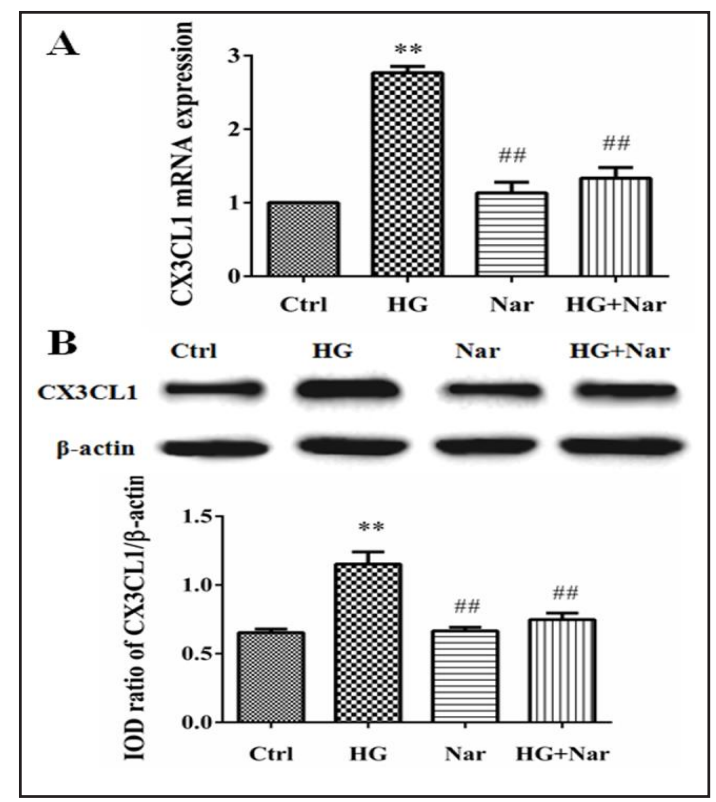

Fig. 4. Effects of naringin on the phosphorylation of AKT in HUVECs at high glucose concentrations. HUVECs had been cultured for 5 days. Cell homogenates were processed by SDS-PAGE electrophoresis and immunoblotting. The phosphorylation of AKT was detected. The image is representative of six independent experiments with similar results. The bar charts show the data from the statistical analysis of the band intensities of activated AKT. Values are the mean \pm SEM of six independent experiments. ${ }^{* *} \mathrm{p}<0.01$ vs. Ctrl group; \#\# $<<0.01$ vs. HG group.

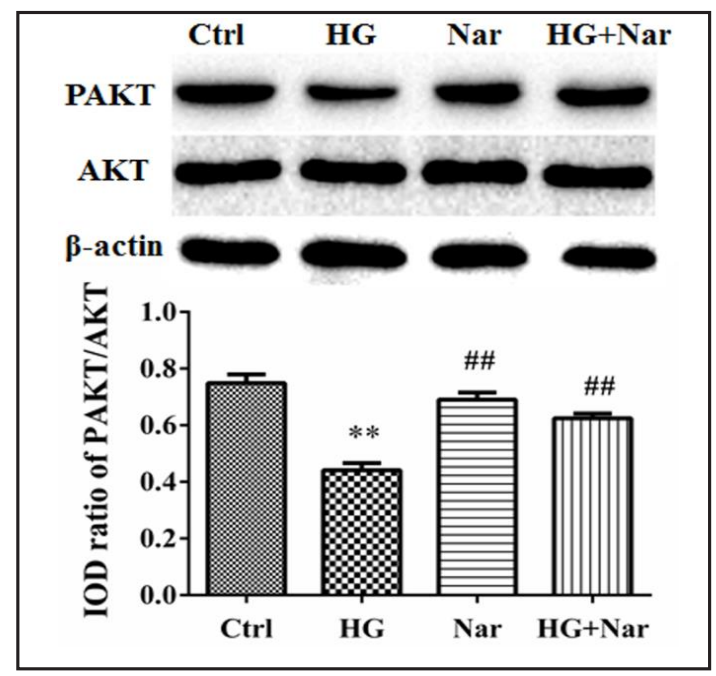

The effect of naringin on PAKT/AKT expression

HUVECs were cultured in control $(5.5 \mathrm{mM})$ or high $(33 \mathrm{mM})$ glucose in the presence or absence of $50 \mu \mathrm{M}$ naringin for 5 days. Phosphorylation (indicative of activation) of AKT was detected by western blotting. Phosphorylation in the high glucose group was reduced 1.7 -fold below the controls $(p<0.01)$. However, naringin rescued the decreased activation of AKT by high glucose $(p<0.01)$. There was no significant difference in AKT protein expression among the four groups, and $\beta$-actin was used as an internal control $\left(\mathrm{F}_{(3,20)}=0.278, p>0.05\right)$ (Fig. 4).

\section{The effects of Naringin on ROS and NO}

To assess the oxidative damage by high glucose on HUVECs and the potential protective effect of naringin, ROS production in HUVECs was examined when they had been cultured with control or high glucose in the absence or presence of naringin $(50 \mu \mathrm{M})$ for 5 days. Intracellular ROS production in HUVECs cultured with high glucose was 1.8-fold higher than in cells cultured with the control medium, whereas naringin reduced high glucose-induced ROS production in HUVECs $(p<0.01)$. NO production was assessed to further elucidate the protective effect of naringin on HUVECs under high glucose conditions. The results showed that NO production in HUVECs cultured with high glucose medium was lower than 
Fig. 5. Effects of naringin on ROS and NO production in HUVECs. Intracellular ROS production was measured after HUVECs were loaded with the ROS fluorescence indicator DCFH-DA $(10 \mu \mathrm{M})$. Data are normalized to ROS production in control HUVECs and expressed as fold of control (A). NO production was measured with a nitrate reductase assay. After different treatments for 5 days, culture media were collected for the measurement of the concentration of NO (B). Data are the mean \pm SEM of nine independent experiments. ${ }^{* *} \mathrm{p}<0.01$ vs. Ctrl group; \#\#p<0.01 vs. HG group.

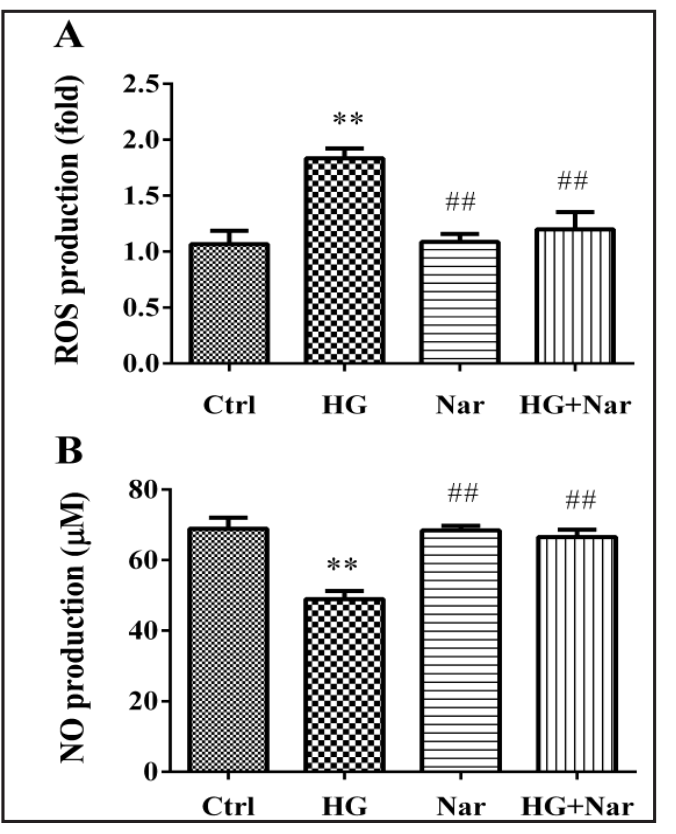

Fig. 6. Effects of naringin on the bioenergetic profile of HUVECs. (A) The oxygen consumption rate (OCR) was determined with the XF analyzer. (B) Basal respiration, (C) ATP production, (D) maximal respiratory and (E) respiratory reserve capacity were all significantly lower in high glucose samples than in controls. Naringin increased the mitochondrial function of HUVECs. Oligo: oligomycin, an ATP synthase blocker; FCCP: carbonyl cyanide 4-(trifluoromethoxy) phenylhydrazone; a/r: antimycin A and rotenone. Data represent the mean \pm SEM of three independent experiments. ${ }^{*} \mathrm{p}<0.05, * * \mathrm{p}<0.01$ vs. Ctrl group; \#p<0.05, \#\#p<0.01 vs. HG group.

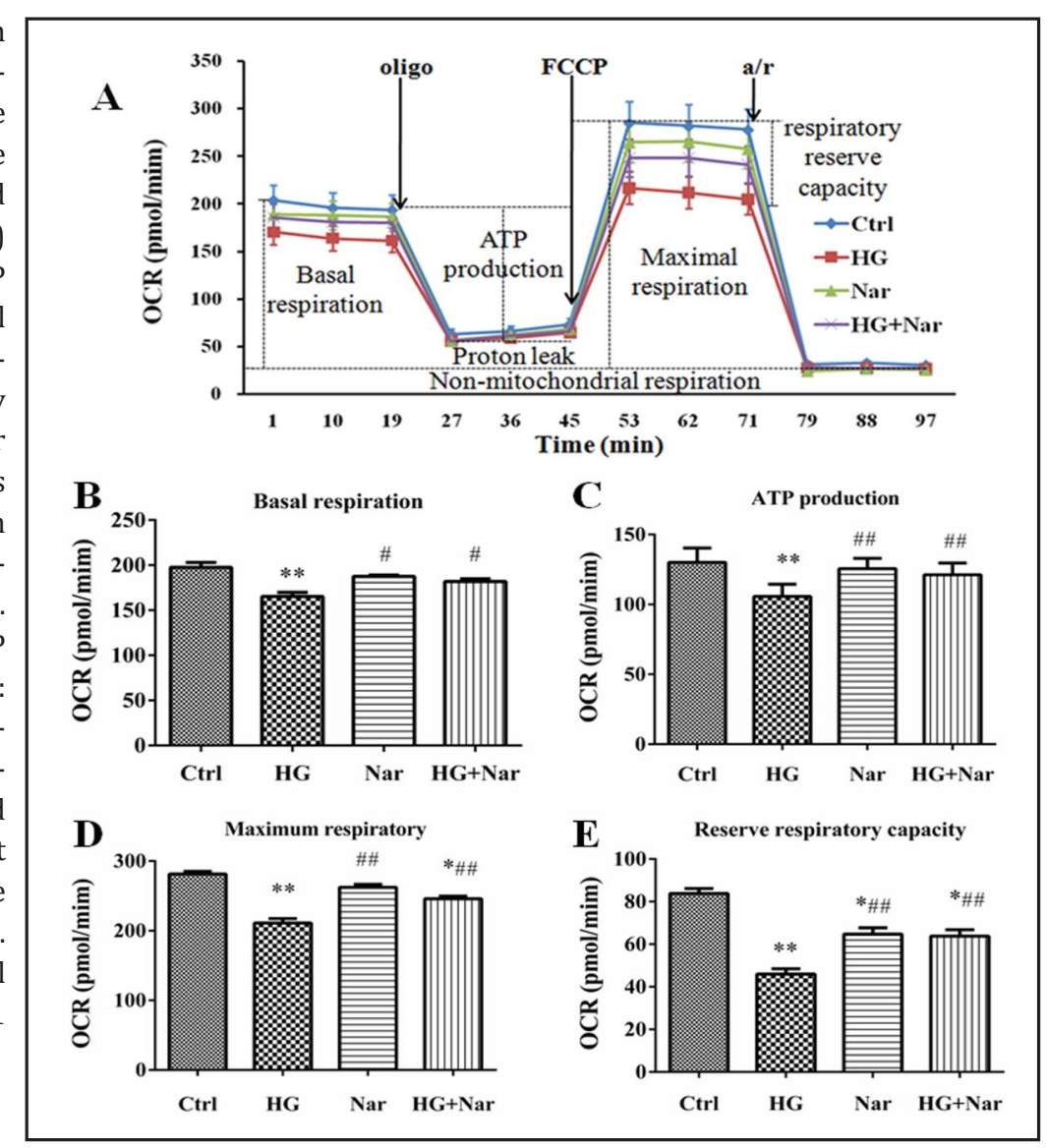

that of controls $(p<0.01)$. However, NO production increased when naringin $(50 \mu \mathrm{M})$ was used with a high glucose treatment $(p<0.01)$. There was no significant difference in ROS $\left(\mathrm{F}_{(2,24)}=0.736, p>0.05\right.$, Fig. 5A) or NO $\left(\mathrm{F}_{(2,24)}=0.659, p>0.05\right.$, Fig. 5B $)$ among the control, control treated with naringin, and the high glucose treated with naringin groups. 


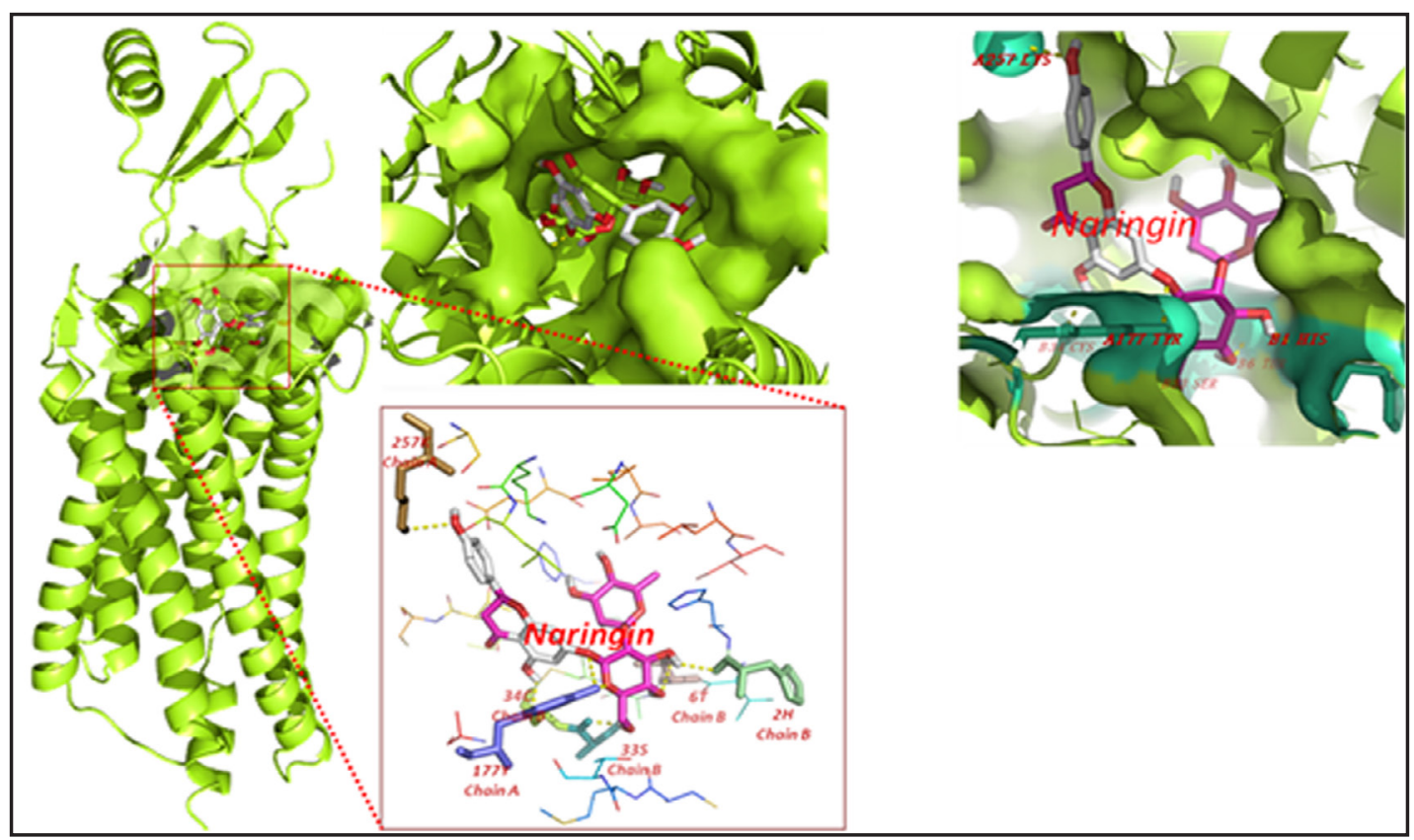

Fig. 7. Molecular docking of naringin on the CX3CL1 protein. Modeling of naringin docking to CX3CL1 was simulated with a computer. Molecular docking poses of naringin are shown in pink within the active site of CX3CL1 (PDB 4XT3). The yellow dashed line indicates a possible hydrogen bond between the connected residues (A257 LYS, B34 CYS, A177 TYR, B34 CYS, B33 SER, B1 HIS and B6 THR) and the ligand. The data show that naringin can interact with the CX3CL1 protein.

\section{Naringin affects the bioenergetic profile of HUVECS}

To evaluate the mitochondrial function of HUVECs, we studied cellular bioenergetics with the Seahorse XF analyzer. Fig. 6A shows that the cellular oxygen consumption rate (OCR) and the response to modifiers of mitochondrial function were markedly lower in cells cultured with high glucose compared to controls $(p<$ 0.01 ). We quantified several bioenergetics parameters with the MitoStress test. The results revealed that high glucose caused a significant reduction in basal respiration (Fig. 6B) and in oxygen consumption due to ATP turnover. Maximal respiration (Fig. 6D) and respiratory reserve capacity (Fig. 6E) were also markedly reduced by high glucose, indicating a depression of mitochondrial activity in hyperglycemic conditions.

Naringin has recently been reported to ameliorate oxidative stress and mitochondrial damage induced by nucleoside reverse transcriptase inhibitors (NRTIs) [20]. We therefore examined whether naringin could improve mitochondrial activity and eventually rescue the negative effect of high glucose on respiration. The exposure of HUVECs to $50 \mu \mathrm{M}$ naringin and $33 \mathrm{mM}$ glucose for 5 days resulted in significant increases in OCR, basal respiration, ATP production, maximal respiration and respiratory reserve capacity (Fig. 6A-E)

Table 1. MOE score of the CX3CL1 protein and naringin $(\mathrm{kcal} / \mathrm{mol})$. The predicted binding affinity is in $\mathrm{kcal} / \mathrm{mol}$ (energy). RMSD values are calculated as related to the best mode and used only movable heavy atoms. Two variants of RMSD metrics are provided: rmsd/lb (RMSD lower bound) and rmsd/ub (RMSD upper bound), which is how the atoms are matched in the distance calculation

\begin{tabular}{cccc} 
Mode & $\begin{array}{c}\text { affinity } \\
(\mathrm{kcal} / \mathrm{mol})\end{array}$ & \multicolumn{2}{c}{$\begin{array}{c}\text { dist from best mode } \\
\mathrm{rmsd} / \mathrm{lb} \mid \mathrm{rmsd} / \mathrm{ub}\end{array}$} \\
\hline 1 & -10.6 & 0.000 & 0.000 \\
2 & -10.0 & 4.032 & 5.353 \\
3 & -9.8 & 3.291 & 4.366 \\
4 & -9.8 & 2.992 & 4.206 \\
5 & -9.4 & 3.379 & 6.085 \\
6 & -9.4 & 2.797 & 7.743 \\
7 & -9.4 & 41.124 & 43.744 \\
8 & -9.3 & 41.004 & 44.272 \\
9 & -9.2 & 2.896 & 9.561 \\
\hline
\end{tabular}




\section{Cellular Physiology Cell Physiol Biochem 2017;42:2540-2551 \\ \begin{tabular}{ll|l} 
DOI: 10.1159/000480215 & O 2017 The Author(s). Published by S. Karger AG, Basel \\
www.karger.com/cpb
\end{tabular} \\ Li et al.: Naringin Protects Huvecs from HG-Induced}

compared to cells cultured in $33 \mathrm{mM}$ glucose. Naringin increased the mitochondrial reserve capacity in cells in response to cellular stress caused by uncoupling. There was no significant difference among the control group, the control treated with naringin group, and high glucose group treated with naringin $\left(\mathrm{F}_{(2,24)}=0.821, p>0.05\right)$.

\section{Molecular docking of naringin on CX3CL1}

The molecular docking of naringin on the CX3CL1 protein was evaluated with AutoDock 4.2. The steps were as follows. Protein molecules were dehydrated and hydrogenated. After calculation of the Gibbs free energy, the pdbqt file of the CX3CL1 protein was produced with the Grid function of ADT Tools toolbar. For the ligand (naringin), the parameter was set by the ADT Tools column, and the pdbqt file of naringin was saved. The docking score of CX3CL1 and naringin (kcal/mol) showed that naringin had a perfect fit for interacting with the CX3CL1 protein (Table 1, Fig. 7).

\section{Discussion}

Diabetes and hyperglycemia are related to significant vascular dysfunction, leading to a large worldwide disease burden. Vascular endothelial dysfunction predisposes diabetic patients to the development of various microvascular and macrovascular complications. In type 2 diabetes mellitus (T2DM) and metabolic syndrome, oxidative stress is increased, which appears to be the basic factor leading to cardiovascular disease, T2DM and diabetic complications [21]. Our results showed that the production of intracellular reactive oxygen species (ROS) was drastically increased when HUVECs had been exposed to high glucose for 5 days. The increased intracellular ROS induced by hyperglycemia is an initial factor that contributes to endothelial injury. The increased intracellular ROS was significantly inhibited by naringin, indicating that naringin may improve the high glucose-induced dysfunction of the endothelium.

Nitric oxide (NO) is one of the most potent vascular protective factors secreted by endothelial cells, and plays a vital role in maintaining vascular tone and other physiological processes of cells [22]. In addition, NO inhibits endothelial cell apoptosis and stimulates endothelial cell proliferation [23]. High glucose concentrations inhibit endothelial cell NO production and affect endothelial cell survival and proliferation [22, 23]. Our data indicated that high concentrations of glucose reduced the production of NO. It is widely accepted that diabetes impairs AKT-eNOS activity. As a result of high blood sugar, hyperlipidemia, oxidative stress and other factors, the endothelial cell PI3K-AKT-eNOS-NO pathway is impaired, and the production of NO and the level of AKT phosphorylation are decreased [24]. Our results also showed that HUVECs exposed to high glucose had lower levels of AKT phosphorylation, less cell viability and reduced proliferation. The decreased production of NO was significantly increased by naringin, demonstrating that naringin may protect the dysfunctional endothelium against high glucose conditions. The reduced activation of AKT was markedly rescued by naringin, which demonstrates that naringin can ameliorate AKT phosphorylation. The suppression of cell viability and proliferation were ameliorated by naringin, indicating that naringin may improve cell viability and proliferation. Naringin significantly relieved the decrease in NO, AKT phosphorylation, cell viability and proliferation in HUVECs induced by chronic high glucose, which indicates that it is protective and antagonizes cell injury.

Type 2 diabetes mellitus is currently considered a chronic inflammatory response. Our previous results from PCR arrays of human endothelial cells showed that several cytokines and chemokines were upregulated in HUVECs after chronic culture with high glucose [7]. CX3CL1 was one of the upregulated chemokines in the PCR array; it is a membrane-bound chemokine that signals through the G protein-coupled receptor CX3CR1 and is involved in the development of diabetic cardiovascular disease. We confirmed the significant upregulation of CX3CL1 in HUVECs cultured with high glucose by qPCR and western blotting. CX3CL1 induces kinase (ERK) and AKT phosphorylation via extracellular signal regulation. 


\section{Cellular Physiology Cell Physiol Biochem 2017;42:2540-2551 \begin{tabular}{ll|l} 
DOI: 10.1159/000480215 & O 2017 The Author(s). Published by S. Karger AG, Basel \\
www.karger.com/cpb
\end{tabular} \\ Li et al.: Naringin Protects Huvecs from HG-Induced}

The inhibition of ERK and PI3K signaling impedes cell proliferation, whereas only PI3K signaling is involved in the anti-apoptotic effect of CX3CL1 [25]. It was noted that CX3CLl and CX3CRl would bind together and then activate and synthesize other adhesion molecules to synergistically enhance adhesion [26]. Our data for the upregulation of CX3CL1 showed that elevated CX3CL1 expression might inhibit cell proliferation and AKT phosphorylation. The CX3CL1 / CX3CR1 axis interacts with several types of cellular adhesion molecules and is considered a significant contributor to the pathogenesis of many inflammatory diseases [27]. The upregulation of CX3CL1 may result from higher ROS generation and can promote inflammatory events. We confirmed the significant increase in the expression of CX3CL1 mRNA and protein in HUVECs cultured with high glucose by qPCR and western blots. Our results also showed that naringin treatment markedly reduced the upregulation of CX3CL1 mRNA and protein under high glucose conditions. The data showed that increased CX3CL1 expression may inhibit cell proliferation and AKT phosphorylation. Our results of the molecular docking of naringin on the CX3CL1 protein indicated that naringin could affect CX3CL1. A higher negative interaction energy indicates a more stable interaction between CX3CL1 and naringin. Naringin binding to CX3CL1 could inhibit the expression of CX3CL1. Therefore, naringin can protect endothelial cells from high glucose-induced inflammatory injury by downregulating CX3CL1, thereby eliminating the inhibition of cell proliferation.

Naringin is a citrus flavonoid found in grape seeds. Flavonoids can interact with intracellular signaling pathways, enhance vascular function, and facilitate synaptic plasticity, in addition to their classical antioxidant properties [28]. Naringin can act as an antioxidant to remove ROS and prevent ROS accumulation and oxidative damage; it can also have an anti-inflammatory, anti-atherosclerotic effect [29]. Our results in the cell mito stress test showed that high glucose significantly decreased the oxygen consumption rate (OCR), which suggests the inhibition of mitochondrial activity. Recent studies have reported that naringin can ameliorate mitochondrial dysfunction [20, 30, 31]. Mitochondrial dysfunction in diabetes mellitus is increasingly being seen as a key mechanism that leads to atherosclerotic complications in diabetes [32]. Our data revealed that naringin rescued the lowered OCR, basal respiration, ATP production, maximal respiration and respiratory reserve capacity in cells exposed to a high glucose concentration. Thus, naringin improved mitochondrial function in HUVECs cultured in high glucose medium.

\section{Conclusion}

In summary, HUVECs cultured with a high concentration of glucose exhibited an increased expression of CX3CL1, which inhibited cell proliferation by reducing AKT phosphorylation. Naringin can interact with the CX3CL1 protein and decrease the expression of CX3CL1 under high glucose conditions. Furthermore, high glucose reduced oxygen consumption in the mitochondrial electron transport chain, which was linked to an increase in mitochondriaderived ROS. Naringin reduced ROS production and ameliorated mitochondrial function. Therefore, naringin protected HUVECs from high glucose as an antioxidant, thereby reducing the expression of CX3CL1 and improving mitochondrial function.

\section{Acknowledgements}

This study was supported by grants from the National Natural Science Foundation of China (81560219, 81200853, 81570735, 31560276, 81360436 and 81360140), and a grant from the young scientist training object of Jiangxi Province (20153BCB23030), and a grant from the Natural Science Foundation of Jiangxi Province (20171BAB205025).

\section{Disclosure Statement}

The authors declare no conflicts of interest.

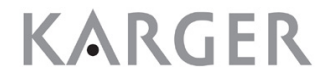




\section{Cellular Physiology Cell Physiol Biochem 2017;42:2540-2551 \begin{tabular}{l|l|l} 
and Biochemistry Published online: August 23, 2017 & $\begin{array}{l}\text { (c) } 2017 \text { The Author(s). Published by S. Karger AG, Basel } \\
\text { www.karger.com/cpb }\end{array}$
\end{tabular}}

Li et al.: Naringin Protects Huvecs from HG-Induced

\section{References}

1 Thomas SR, Witting PK, Drummond GR: Redox control of endothelial function and dysfunction: molecular mechanisms and therapeutic opportunities. Antioxid Redox Signal 2008;10:1713-1765.

-2 Li J, Li J, Wei T, Li J: Down-Regulation of MicroRNA-137 Improves High Glucose-Induced Oxidative Stress Injury in Human Umbilical Vein Endothelial Cells by Up-Regulation of AMPKalpha1. Cell Physiol Biochem 2016;39:847-859.

-3 Giugliano D, Ceriello A, Paolisso G: Oxidative stress and diabetic vascular complications. Diabetes Care 1996;19:257-267.

-4 Zhong ZY, Tang Y: Upregulation of Periostin Prevents High Glucose-Induced Mitochondrial Apoptosis in Human Umbilical Vein Endothelial Cells via Activation of Nrf2/HO-1 Signaling. Cell Physiol Biochem 2016;39:71-80.

-5 Czajka A, Malik AN: Hyperglycemia induced damage to mitochondrial respiration in renal mesangial and tubular cells: Implications for diabetic nephropathy. Redox Biol 2016;10:100-107.

-6 Avogaro A, Fadini GP, Gallo A, Pagnin E, de Kreutzenberg S: Endothelial dysfunction in type 2 diabetes mellitus. Nutr Metab Cardiovasc Dis 2006;16 Suppl 1:S39-45.

7 Li G, Zhu G, Gao Y, Xiao W, Xu H, Liu S, Tu G, Peng H, Zheng C, Liang S, Li G: Neferine inhibits the upregulation of CCL5 and CCR5 in vascular endothelial cells during chronic high glucose treatment. Inflammation 2013;36:300-308.

8 Flierl U, Bauersachs J, Schafer A: Modulation of platelet and monocyte function by the chemokine fractalkine (CX3 CL1) in cardiovascular disease. Eur J Clin Invest 2015;45:624-633.

-9 Chen F, Zhang N, Ma X, Huang T, Shao Y, Wu C, Wang Q: Naringin Alleviates Diabetic Kidney Disease through Inhibiting Oxidative Stress and Inflammatory Reaction. PLoS One 2015;10:e0143868.

$\checkmark 10$ Chtourou Y, Aouey B, Aroui S, Kebieche M, Fetoui H: Anti-apoptotic and anti-inflammatory effects of naringin on cisplatin-induced renal injury in the rat. Chem Biol Interact 2016;243:1-9.

$\checkmark 11$ Adebiyi A0, Adebiyi 00, Owira PM: Naringin Mitigates Cardiac Hypertrophy by Reducing Oxidative Stress and Inactivating c-Jun Nuclear Kinase-1 Protein in Type I Diabetes. J Cardiovasc Pharmacol 2016;67:136144.

12 Jung UJ, Lee MK, Jeong KS, Choi MS: The hypoglycemic effects of hesperidin and naringin are partly mediated by hepatic glucose-regulating enzymes in C57BL/KsJ-db/db mice. J Nutr 2004;134:2499-2503.

13 Bharti S, Rani N, Krishnamurthy B, Arya DS: Preclinical evidence for the pharmacological actions of naringin: a review. Planta Med 2014;80:437-451.

14 Liu X, Liu M, Mo Y, Peng H, Gong J, Li Z, Chen J, Xie J: Naringin ameliorates cognitive deficits in streptozotocin-induced diabetic rats. Iran J Basic Med Sci 2016;19:417-422.

15 Dranka BP, Benavides GA, Diers AR, Giordano S, Zelickson BR, Reily C, Zou L, Chatham JC, Hill BG, Zhang J, Landar A, Darley-Usmar VM: Assessing bioenergetic function in response to oxidative stress by metabolic profiling. Free Radic Biol Med 2011;51:1621-1635.

16 Trudeau K, Molina AJ, Roy S: High glucose induces mitochondrial morphology and metabolic changes in retinal pericytes. Invest Ophthalmol Vis Sci 2011;52:8657-8664.

17 Foresti R, Bucolo C, Platania CM, Drago F, Dubois-Rande JL, Motterlini R: Nrf2 activators modulate oxidative stress responses and bioenergetic profiles of human retinal epithelial cells cultured in normal or high glucose conditions. Pharmacol Res 2015;99:296-307.

-18 Trott 0, Olson AJ: AutoDock Vina: improving the speed and accuracy of docking with a new scoring function, efficient optimization, and multithreading. J Comput Chem 2010;31:455-461.

-19 Burg JS, Ingram JR, Venkatakrishnan AJ, Jude KM, Dukkipati A, Feinberg EN, Angelini A, Waghray D, Dror RO, Ploegh HL, Garcia KC: Structural biology. Structural basis for chemokine recognition and activation of a viral G protein-coupled receptor. Science 2015;347:1113-1117.

20 Oluwafeyisetan A, Olubunmi A, Peter O: Naringin Ameliorates HIV-1 Nucleoside Reverse Transcriptase Inhibitors- Induced Mitochondrial Toxicity. Curr HIV Res 2016;14:506-516.

21 Tangvarasittichai S: Oxidative stress, insulin resistance, dyslipidemia and type 2 diabetes mellitus. World J Diabetes 2015;6:456-480.

22 Wang LP, Jiang Y, Yang H, Peng C, Zhang C, Tao X, Xie HH: Combination Therapy of Nifedipine and Sulphonylureas Exhibits a Mutual Antagonistic Effect on the Endothelial Cell Dysfunction Induced by Hyperglycemia Linked to Vascular Disease. Cell Physiol Biochem 2016;38:2337-2347. 


\section{Cellular Physiology Cell Physiol Biochem 2017;42:2540-2551

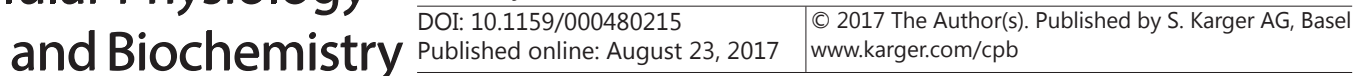 \\ Li et al.: Naringin Protects Huvecs from HG-Induced}

23 Wang F, Pu C, Zhou P, Wang P, Liang D, Wang Q, Hu Y, Li B, Hao X: Cinnamaldehyde prevents endothelial dysfunction induced by high glucose by activating Nrf2. Cell Physiol Biochem 2015;36:315-324.

-24 Li X, Xing W, Wang Y, Mi C, Zhang Z, Ma H, Zhang H, Gao F: Upregulation of caveolin-1 contributes to aggravated high-salt diet-induced endothelial dysfunction and hypertension in type 1 diabetic rats. Life Sci 2014;113:31-39.

25 White GE, Tan TC, John AE, Whatling C, McPheat WL, Greaves DR: Fractalkine has anti-apoptotic and proliferative effects on human vascular smooth muscle cells via epidermal growth factor receptor signalling. Cardiovasc Res 2010;85:825-835.

-26 Fujita M, Takada YK, Takada Y: The chemokine fractalkine can activate integrins without CX3CR1 through direct binding to a ligand-binding site distinct from the classical RGD-binding site. PLoS One 2014;9:e96372.

-27 Poniatowski LA, Wojdasiewicz P, Krawczyk M, Szukiewicz D, Gasik R, Kubaszewski L, KurkowskaJastrzebska I: Analysis of the Role of CX3CL1 (Fractalkine) and Its Receptor CX3CR1 in Traumatic Brain and Spinal Cord Injury: Insight into Recent Advances in Actions of Neurochemokine Agents. Mol Neurobiol 2017;54:2167-2188.

28 Ramalingayya GV, Nampoothiri M, Nayak PG, Kishore A, Shenoy RR, Mallikarjuna Rao C, Nandakumar K: Naringin and Rutin Alleviates Episodic Memory Deficits in Two Differentially Challenged Object Recognition Tasks. Pharmacogn Mag 2016;12:S63-70.

29 Buachan P, Chularojmontri L, Wattanapitayakul SK: Selected activities of Citrus maxima Merr. fruits on human endothelial cells: enhancing cell migration and delaying cellular aging. Nutrients 2014;6:16181634 .

-30 Kulasekaran G, Ganapasam S: Neuroprotective efficacy of naringin on 3-nitropropionic acid-induced mitochondrial dysfunction through the modulation of Nrf2 signaling pathway in PC12 cells. Mol Cell Biochem 2015;409:199-211.

-31 Huang H, Wu K, You Q, Huang R, Li S, Wu K: Naringin inhibits high glucose-induced cardiomyocyte apoptosis by attenuating mitochondrial dysfunction and modulating the activation of the p38 signaling pathway. Int J Mol Med 2013;32:396-402.

-32 Joshi MS, Williams D, Horlock D, Samarasinghe T, Andrews KL, Jefferis AM, Berger PJ, Chin-Dusting JP, Kaye DM: Role of mitochondrial dysfunction in hyperglycaemia-induced coronary microvascular dysfunction: Protective role of resveratrol. Diab Vasc Dis Res 2015;12:208-216. 